\title{
Clinical Studies of Lactoferrin in Neonates and Infants: An Update
}

\author{
Paolo Manzoni
}

Keywords: human milk, lactoferrin, infections, neonate, preterm

$\mathbf{H}$ UMAN MILK CONTAINS many bioactive factors that support organ development, contribute to the maturation of the immune system, and provide protection against neonatal infection and diseases, such as bronchopulmonary disease, retinopathy of prematurity, and necrotizing enterocolitis (NEC). ${ }^{1}$ Lactoferrin is a glycoprotein found in human milk that is associated with a number of potentially important health benefits. ${ }^{2}$ Concentrations of lactoferrin are highest in colostrum and abruptly decrease in more mature milk; this decrease occurs in all mammals (Fig. 1). Bovine and human lactoferrin share strong $(77 \%)$ sequence homology ${ }^{3}$ and the same antimicrobial peptide ( $n$-lactoferricin). Lactoferrin demonstrates antimicrobial activity through several direct and indirect mechanisms, such as sequestering iron (a substrate for pathogens) and inhibiting the growth, adhesion, translocation, and virulence of pathogens. ${ }^{4-6}$ Lactoferrin has also been shown to stimulate cells involved in innate and acquired immunity. ${ }^{7}$ Data from preclinical studies suggest lactoferrin is a key modulator of intestinal epithelium development. Lactoferrin supplementation was shown to improve intestinal mucosal structure and increase villus height and crypt proliferation in mice and piglets. ${ }^{8,9}$ A study of the in vitro effects of human and bovine lactoferrin in enteric Caco 2 cells showed lactoferrin had a concentration-dependent trophic effect on enterocytes; higher concentrations resulted in faster enterocyte proliferation, whereas lower concentrations resulted in faster enterocyte differentiation. ${ }^{10}$ Lactoferrin may also play a role in establishing the neonatal gut microbiota. A study in 48 term and preterm infants found that the amount of fecal bifidobacteria and lactobacilli was significantly associated with fecal lactoferrin concentrations 3 days after delivery, suggesting lactoferrin may promote the growth of beneficial gut microbiota. ${ }^{11}$

Multiple clinical studies have suggested a number of potentially favorable biologic effects associated with lactoferrin in infants and children. ${ }^{12-16}$ The first randomized controlled trial (RCT) to assess lactoferrin supplementation in neonates reported a significant reduction in the incidence of late-onset sepsis with bovine lactoferrin compared with placebo in very low birth weight preterm infants (Fig. 2). ${ }^{3}$ A secondary analysis of the study data found that bovine lactoferrin supplementation reduced the incidence of fungal infection versus placebo $(0.8 \%$ versus $5.4 \% ; p=0.009)$, yet there was no effect on fungal colonization in the gut. ${ }^{17}$ Another RCT also reported a lower incidence of late-onset sepsis with bovine lactoferrin supplementation versus placebo in very low birth weight infants $(20 \%$ versus $37.5 \% ; p=0.047) .{ }^{18}$ Similar positive effects on sepsis were reported in other RCTs evaluating bovine lactoferrin supplementation in very low birth weight neonates; however, the studies were limited by small sample size and underpowered. ${ }^{19,20}$ A separate RCT in very low birth weight infants showed that bovine lactoferrin supplementation reduced the incidence of NEC and/or mortality compared with placebo $(4.0 \%$ versus $10.1 \% ; p=0.008) .{ }^{21}$ The only study evaluating human lactoferrin supplementation in

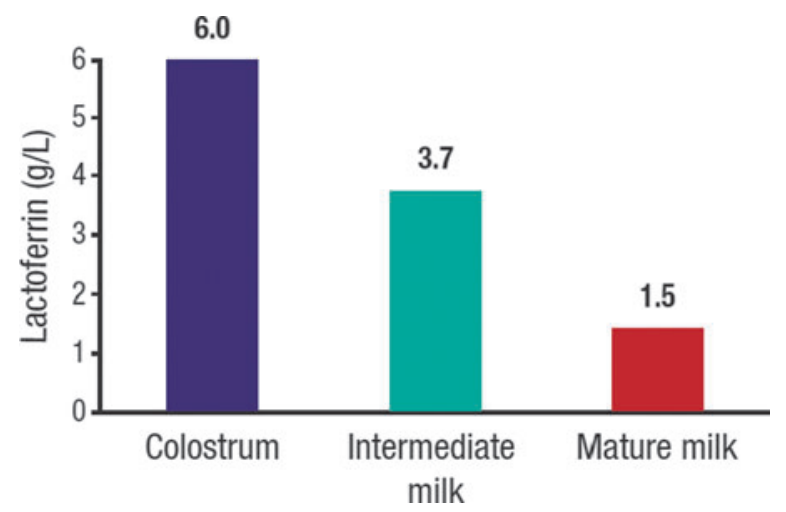

FIG. 1. Lactoferrin concentrations decrease in mature human milk versus colostrum.

Division of Pediatrics and Neonatology, Degli Infermi Hospital, Biella, Italy.

(C) Paolo Manzoni, 2019; Published by Mary Ann Liebert, Inc. This Open Access article is distributed under the terms of the Creative Commons License (http://creativecommons.org/licenses/by/4.0), which permits unrestricted use, distribution, and reproduction in any medium, provided the original work is properly cited. 
A

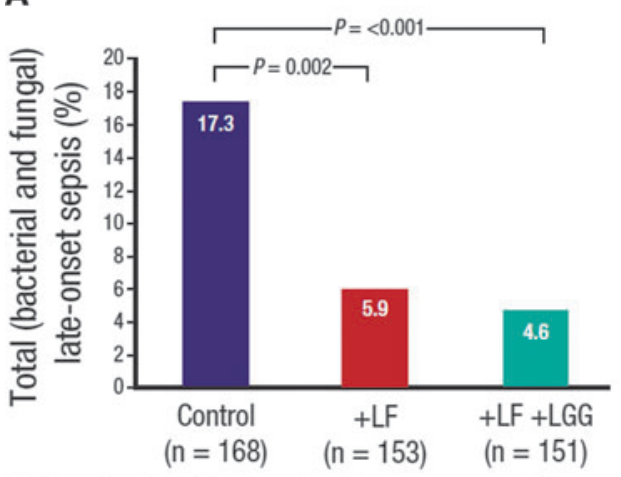

B

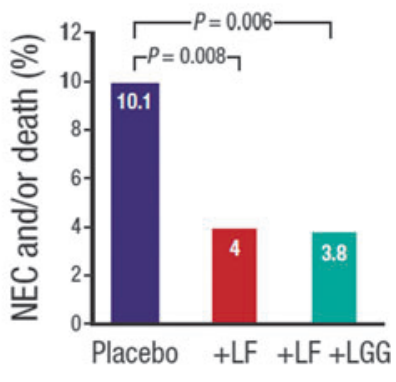

FIG. 2. Supplemental lactoferrin reduced incidence of (A) late-onset sepsis and (B) NEC and/or mortality in very low birth weight infants. ${ }^{3,21} \mathrm{LF}$, lactoferrin; LGG, Lactobacillus rhamnosus GG; NEC, necrotizing enterocolitis.

LF, lactoferrin; LGG, Lactobacillus rhamnosus GG.

very low birth weight infants reported lower rates of hospitalacquired infections with lactoferrin versus placebo $(17 \%$ versus $33 \% ; p<0.04)$, but the study was underpowered to identify significant differences in outcomes between the two groups. ${ }^{22}$

A recent Cochrane review that included six RCTs evaluating lactoferrin supplementation in preterm neonates concluded that lactoferrin supplementation, with or without probiotics, decreased late-onset sepsis (risk ratio [RR], 0.59; $p=0.008)$ and NEC (RR, 0.40; $p=0.02$ ) with no adverse effects, but the available evidence was graded as "low" or "low to moderate" quality. ${ }^{23}$ Further studies are needed to compare the different types of lactoferrin (human or bovine) to determine optimal dosing regimens and to assess longterm outcomes. Data from the recently completed ELFIN (enteral lactoferrin in neonates; $N=2,200$ ) and LIFT (lactoferrin infant feeding trial; $N=1,500)$ studies will help clarify the potential benefits of lactoferrin supplementation in preterm infants.

\section{Disclosure Statement}

P.M. received speaker's fee from Prolacta.

\section{References}

1. Ballard O, Morrow AL. Human milk composition: Nutrients and bioactive factors. Pediatr Clin North Am 2013;60:49-74.

2. Kanwar JR, Roy K, Patel Y, et al. Multifunctional iron bound lactoferrin and nanomedicinal approaches to enhance its bioactive functions. Molecules 2015;20: 9703-9731.

3. Manzoni P, Rinaldi M, Cattani S, et al. Bovine lactoferrin supplementation for prevention of late-onset sepsis in very low-birth-weight neonates: A randomized trial. JAMA 2009; 302:1421-1428.

4. Ochoa TJ, Cleary TG. Effect of lactoferrin on enteric pathogens. Biochimie 2009;91:30-34.

5. Teraguchi S, Shin K, Ogata T, et al. Orally administered bovine lactoferrin inhibits bacterial translocation in mice fed bovine milk. Appl Environ Microbiol 1995;61:41314134.

6. Reznikov EA, Comstock SS, Hoeflinger JL, et al. Dietary bovine lactoferrin reduces Staphylococcus aureus in the tissues and modulates the immune response in piglets systemically infected with S. aureus. Curr Dev Nutr 2017;2:nzy001.

7. Shan T, Wang Y, Wang Y, et al. Effect of dietary lactoferrin on the immune functions and serum iron level of weanling piglets. J Anim Sci 2007;85:2140-2146.

8. Reznikov EA, Comstock SS, Yi C, et al. Dietary bovine lactoferrin increases intestinal cell proliferation in neonatal piglets. J Nutr 2014;144:1401-1408.

9. Li Q, Hu W, Zhao J, et al. Supplementation transgenic cow's milk containing recombinant human lactoferrin enhances systematic and intestinal immune responses in piglets. Mol Biol Rep 2014;41:2119-2128.

10. Buccigrossi V, de Marco G, Bruzzese E, et al. Lactoferrin induces concentration-dependent functional modulation of intestinal proliferation and differentiation. Pediatr Res 2007;61:410-414.

11. Mastromarino P, Capobianco D, Campagna G, et al. Correlation between lactoferrin and beneficial microbiota in breast milk and infant's feces. Biometals 2014;27: 1077-1086.

12. King JC Jr, Cummings GE, Guo N, et al. A double-blind, placebo-controlled, pilot study of bovine lactoferrin supplementation in bottle-fed infants. J Pediatr Gastroenterol Nutr 2007;44:245-251.

13. Egashira M, Takayanagi $\mathrm{T}$, Moriuchi $\mathrm{M}$, et al. Does daily intake of bovine lactoferrin-containing products ameliorate rotaviral gastroenteritis? Acta Paediatr 2007;96:1242-1244.

14. Ochoa TJ, Chea-Woo E, Campos M, et al. Impact of lactoferrin supplementation on growth and prevalence of Giardia colonization in children. Clin Infect Dis 2008;46: 1881-1883.

15. Zuccotti GV, Trabattoni D, Morelli M, et al. Immune modulation by lactoferrin and curcumin in children with recurrent respiratory infections. J Biol Regul Homeost Agents 2009;23:119-123.

16. Ochoa TJ, Chea-Woo E, Baiocchi N, et al. Randomized double-blind controlled trial of bovine lactoferrin for prevention of diarrhea in children. J Pediatr 2013;162: 349-356.

17. Manzoni P, Stolfi I, Messner H, et al. Bovine lactoferrin prevents invasive fungal infections in very low birth weight infants: A randomized controlled trial. Pediatrics 2012; 129:116-123.

18. Ochoa TJ, Zegarra J, Cam L, et al. Randomized controlled trial of lactoferrin for prevention of sepsis in Peruvian neonates less than 2500 g. Pediatr Infect Dis $J$ 2015;34:571-576. 
19. Akin IM, Atasay B, Dogu F, et al. Oral lactoferrin to prevent nosocomial sepsis and necrotizing enterocolitis of premature neonates and effect on T-regulatory cells. Am J Perinatol 2014;31:1111-1120.

20. Kaur G, Gathwala G. Efficacy of bovine lactoferrin supplementation in preventing late-onset sepsis in low birth weight neonates: A randomized placebo-controlled clinical trial. J Trop Pediatr 2015;61:370-376.

21. Manzoni P, Meyer M, Stolfi I, et al. Bovine lactoferrin supplementation for prevention of necrotizing enterocolitis in very-low-birth-weight neonates: A randomized clinical trial. Early Hum Dev 2014;90:S60-S65.

22. Sherman MP, Adamkin DH, Niklas V, et al. Randomized controlled trial of talactoferrin oral solution in preterm infants. J Pediatr 2016;175:68-73.e3.
23. Pammi M, Suresh G. Enteral lactoferrin supplementation for prevention of sepsis and necrotizing enterocolitis in preterm infants. Cochrane Database Syst Rev 2017;6: CD007137.

Address correspondence to:

Paolo Manzoni, MD, PhD

Division of Pediatrics and Neonatology

Degli Infermi Hospital

Via dei Ponderanesi 2

31785 Ponderano (BI)

Italy

E-mail: paolomanzoni@hotmail.com 